\title{
A small-gain type stability criterion for large scale networks of ISS systems
}

\author{
Sergey Dashkovskiy
}

\author{
Björn Sebastian Rüffer
}

\author{
Fabian R. Wirth
}

\begin{abstract}
We provide a generalized version of the nonlinear small-gain theorem for the case of more than two coupled inputto-state stable systems. For this result the interconnection gains are described in a nonlinear gain matrix and the small gain condition requires bounds on the image of this gain matrix. The condition may be interpreted as a nonlinear generalization of the requirement that the spectral radius of the gain matrix is less than one. We give some interpretations of the condition in special cases covering linear gains and linear systems.
\end{abstract}

Index Terms-Interconnected systems, stability, ISS, smallgain theorem, gain matrix, large-scale systems

\section{INTRODUCTION}

Stability is one of the fundamental concepts in the analysis and design of nonlinear dynamical systems. The notions of input-to-state stability (ISS) and nonlinear gains has proved to be an efficient tool for the qualitative description of the stability of nonlinear input systems. In this paper we consider nonlinear systems consisting of several interconnected subsystems and investigate their stability properties.

There are different equivalent formulations of ISS: In terms of $\mathcal{K} \mathcal{L}$ and $\mathcal{K}_{\infty}$ functions (see below), via Lyapunov functions, as an asymptotic stability property combined with asymptotic gains, and others, see [11]. A more quantitative but equivalent formulation, which captures the long term dynamic behavior of the system, is the notion of input-tostate dynamical stability (ISDS), see [2].

To analyze the stability of two ISS systems in a feedback interconnection there are known small-gain theorems providing the restrictions on the gains of two ISS subsystems to ensure that the feedback interconnection is ISS, cf. [5], [4], [7], [2]. These results state that if the composition of the gain functions $\gamma_{1}(\cdot), \gamma_{2}(\cdot)$ of ISS subsystems is small enough, then the whole system is ISS. In case of linear gain functions this condition reads

$$
\gamma_{1} \circ \gamma_{2}<\mathrm{Id}
$$

we discuss the general condition in Section IV.

The papers [5], [4], [2] have different approaches to formulate and prove the sufficiency of the small-gain condition: In [5] the proof is based on the properties of $\mathcal{K L}$ and $\mathcal{K}_{\infty}$ functions (see below for the definition). The result in that paper also covers practical ISS results, which we do not treat here. An ISS-Lyapunov function for the feedback system is constructed in [4] as some combination of the

S. Dashkovskiy and B. S. Rüffer are with the Zentrum für Technomathematik, University of Bremen, Germany, $\{$ dsn, rueffer $\}$ emath. uni-bremen. de

F. R. Wirth is with the Hamilton Institute, NUI Maynooth, Maynooth, Co. Kildare, Ireland, fabian.wirthenuim.ie corresponding ISS-Lyapunov functions of both subsystems. The key assumption in the proof of that paper is that the gains are already provided in terms of the Lyapunov functions. The proof of the small-gain theorem in [2] is based on the ISDS property. As a further result on system interconnections we note that it is well known, that a cascade of two ISS systems is ISS, see, e.g., [9] or [10]. These results will turn out to be special cases of the main result of this paper. In this paper, we choose an approach using estimates involving $\mathcal{K} \mathcal{L}$ and $\mathcal{K}_{\infty}$-functions to prove an ISS stability result for general interconnected systems satisfying a generalized small-gain condition. Although we believe that the approach should be amenable to the explicit construction of a Lyapunov function given Lyapunov functions for the subsystems, we have not yet been able to achieve this goal.

We note, that in [12] small-gain theorems for general interconnected systems with linear gains can be found, where the gains are taken with respect to any $p$-norm, $p \in[1, \infty]$, on the interconnections. These results are of the form that the spectral radius of a gain matrix should be less than one to conclude stability. The result of this paper may be regarded as a nonlinear generalization in the same spirit. We quote also [8] for some other stability results on large-scale systems.

In this paper we consider a system which consists of three or more ISS subsystems. We wish to provide conditions by which the stability question of the overall system can be reduced to the consideration of the stability of the subsystems. While this can be approached by repeated application of the cascade property and the known small-gain theorem, in general this can be a cumbersome problem and it is by no means obvious in which order the subsystems have to be chosen to proceed in such an iterative manner. Even worse, this may not always work, see the motivating example below. Hence an extension of the known small-gain theorem to larger interconnections is needed. In this paper we obtain this extension for the general case. Further, we show how to calculate the gain matrix for linear systems and give some interpretation of our result.

The paper is organized as follows. In Section II we introduce notation and necessary concepts. In particular, we will need some basic properties of the positive orthant $\mathbb{R}_{+}^{n}$ interpreted as a lattice. Also we give an example to motivate the problem, which we state there. In Section III we prove the main result, which generalizes the known small-gain theorem, and consider some special cases (linear gains, linear systems). In Section IV the "small-gain condition" of the main result is discussed and we show in which way it may be interpreted as an extension of the linear condition that 
the spectral radius of the gain matrix is less than one. We conclude with Section V.

\section{Motivation AND PROBLEM DESCRIPTION}

a) Notation: In the following $\mathbb{R}_{+}$denotes the interval $[0, \infty)$. By $x^{T}$ we denote the transpose of a vector $x$.

Definition 1: (i) A function $\gamma: \mathbb{R}_{+} \rightarrow \mathbb{R}_{+}$is said to be of class $\mathcal{K}$ if it is continuous, increasing and $\gamma(0)=0$. It is of class $\mathcal{K}_{\infty}$ if, in addition, it is proper, i.e., unbounded.

(ii) A function $\beta: \mathbb{R}_{+} \times \mathbb{R}_{+} \rightarrow \mathbb{R}_{+}$is said to be of class $\mathcal{K L}$ if, for each fixed $t$, the function $\beta(\cdot, t)$ is of class $\mathcal{K}$ and, for each fixed $s$, the function $\beta(s, \cdot)$ is non-increasing and tends to zero at infinity.

Let $|\cdot|$ denote some norm in $\mathbb{R}^{n},\|\cdot\|$ refers to the supremum or essential supremum norm for essentially bounded functions defined on $\mathbb{R}_{+}$.

Definition 2: Consider a system

$$
\dot{x}=f(x, u), x \in \mathbb{R}^{n}, u \in \mathbb{R}^{m}
$$

such that for all initial values $x_{0}$ and all essentially bounded inputs $u(\cdot)$ unique solutions exist for all positive times. The system is called input to state stable (ISS), if there exist functions $\beta$ of class $\mathcal{K} \mathcal{L}$ and $\gamma$ of class $\mathcal{K}$, such that for the solution $\xi\left(t ; x_{0}, u\right)$ starting at $x_{0}$ and applying $u$

$$
\left|\xi\left(t ; x_{0}, u\right)\right| \leq \beta\left(\left|x_{0}\right|, t\right)+\gamma(\|u\|)
$$

holds for all $t \geq 0, x_{0} \in \mathbb{R}^{n}, u$ essentially bounded.

b) Motivating example: Consider a basic network consisting of three coupled subsystems $\Sigma_{1}, \Sigma_{2}$ and $\Sigma_{3}$, each having the ISS property with well defined gains between individual subsystems.

The system is depicted in Figure 1 which is to be interpreted as follows: $u$ is some external input, each state $x_{i}$ of system $\Sigma_{i}$ is fed into another subsystem $\Sigma_{j}$ with the linear gain written at the edge connecting them. E.g., for $\Sigma_{2}$ the ISS property reads as follows: There is a bound for the state $x_{2}$ of $\Sigma_{2}$ of the form

$$
\left|x_{2}(t)\right| \leq \beta\left(x_{2}(0), t\right)+\varepsilon\left\|x_{1}\right\|_{[0, t]}+\frac{2}{3}\left\|x_{3}\right\|_{[0, t]},
$$

and similarly for the other systems. The weighted adjacency matrix of this network is

$$
G=\left[\begin{array}{ccc}
0 & \frac{2}{3} & 0 \\
\varepsilon & 0 & \frac{2}{3} \\
2 & \varepsilon & 0
\end{array}\right]
$$

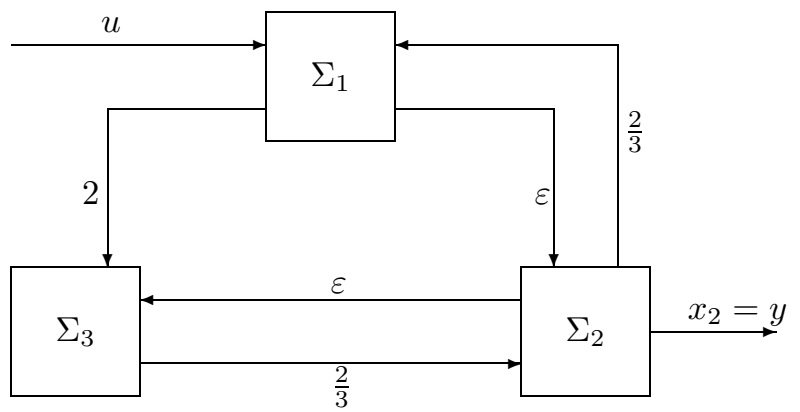

Fig. 1. Example of a feedback network of three subsystems. where we forget about external inputs and edges "leaving" the network. The $(i, j)^{\text {th }}$ entry of $G$ denotes the gain that station $i$ receives from the station $j$. This matrix is called the (linear) gain matrix of the network.

First we consider the case when $\varepsilon=0$, that is, we have a ring network. Intuitively clear, this ring is stable, since the small-gain condition $2 \cdot \frac{2}{3} \cdot \frac{2}{3}=\frac{8}{9}<1$ is met. Indeed, this is the exactly the condition of Corollary 7 .

Now, if we rigorously apply the known small-gain theorem and the cascade rule in an iterative manner, then we always end up at a point, where we cannot simplify the network using the small-gain theorem and hence we are not able to deduce input-to-state stability of the overall network.

Second, what happens, if $\varepsilon>0$ ? Rigorous application of the classical results as before, for example utilizing the small-gain theorem to combine subsystems $\Sigma_{1}$ and $\Sigma_{2}$ to a new subsystem $\tilde{\Sigma}_{12}$ as in Figure 2 (here we assumed that $\varepsilon<\frac{3}{2}$ ), leads to the same situation as before.

In this paper we prove an ISS criterion for such networks. For the above example it reads as follows: The network is ISS provided that the spectral radius $\rho(G)$ of the gain matrix $G$ is strictly less than one. In this particular case one can compute that the network will remain stable at least for feedback disturbances with a linear gain $\varepsilon<\frac{1}{12}$.

c) Additional Preliminaries: For $x, y \in \mathbb{R}^{n}$, we use the following notation

$$
\begin{aligned}
& x \geq y \Longleftrightarrow x_{i} \geq y_{i}, i=1, \ldots, n, \\
& x>y \Longleftrightarrow x_{i}>y_{i}, i=1, \ldots, n,
\end{aligned}
$$

and $x \geq y$ means negation of $x \geq y$. By $\mathbb{R}_{+}^{n}$ we denote $\left\{x \in \mathbb{R}^{n}: x \geq 0\right\}$. The space ( $\mathbb{R}_{+}^{n}$, sup, inf) is a lattice, with inf denoting infimum and sup denoting supremum with respect to the order $\geq$ we have just defined. The upper limit for bounded functions $s: \mathbb{R}_{+} \rightarrow \mathbb{R}_{+}^{n}$ can be defined by

$$
\limsup _{t \rightarrow \infty} s(t):=\inf _{t \geq 0} \sup _{\tau \geq t} s(\tau)
$$

For functions $v: \mathbb{R}_{+} \rightarrow \mathbb{R}^{m}$ we define its restriction to the interval $\left[s_{1}, s_{2}\right]$ by

$$
v_{\left[s_{1}, s_{2}\right]}(t):= \begin{cases}v(t) & \text { if } t \in\left[s_{1}, s_{2}\right] \\ 0 & \text { else. }\end{cases}
$$

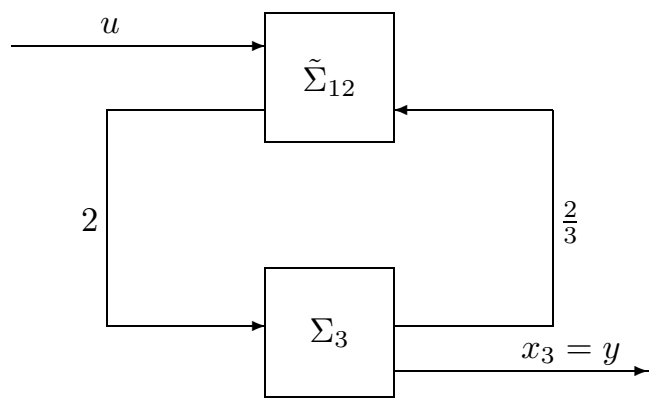

Fig. 2. Reduced network if $\varepsilon<\frac{3}{2}$. 
For functions $x: \mathbb{R}_{+} \rightarrow \mathbb{R}^{N_{1}+\ldots+N_{n}}$ and times $0 \leq t_{1} \leq t_{2}$ we define

$$
\left\|x_{\left[t_{1}, t_{2}\right]}\right\|:=\left(\begin{array}{c}
\left\|x_{1,\left[t_{1}, t_{2}\right]}\right\| \\
\vdots \\
\left\|x_{n,\left[t_{1}, t_{2}\right]}\right\|
\end{array}\right) .
$$

We will need the following lemma. The proof is not difficult and thus omitted for reasons of space.

Lemma 3: Let $s: \mathbb{R}_{+} \rightarrow \mathbb{R}_{+}^{n}$ be continuous and bounded. Then

$$
\limsup _{t \rightarrow \infty} s(t)=\limsup _{t \rightarrow \infty}\left\|s_{[t / 2, \infty)}\right\| .
$$

Before we introduce the ISS condition for interconnected systems let us briefly discuss an equivalent formulation of ISS. A system

$$
\dot{x}=f(x, u),
$$

with $f: \mathbb{R}^{n_{1}+n_{2}} \rightarrow \mathbb{R}^{n_{1}}$ continuous and Lipschitz in $x$ uniformly with respect to $u$, is said to have the asymptotic gain property (AG), if there exists a function $\gamma_{A G} \in \mathcal{K}_{\infty}$ such that for all initial values $x_{0} \in \mathbb{R}^{n_{1}}$ and all essentially bounded control functions $u(\cdot)$

$$
\limsup _{t \geq 0}\left|\xi\left(t ; x_{0}, u\right)\right| \leq \gamma_{A G}(\|u\|) .
$$

The asymptotic gain property states, that every trajectory must ultimately stay not far from zero, depending on the magnitude of $\|u\|$.

The system (5) is said to be globally asymptotically stable at zero (0-GAS), if there exists a $\beta_{G A S} \in \mathcal{K} \mathcal{L}$, such that for all initial conditions $x_{0} \in \mathbb{R}^{n_{1}}$

$$
\left|\xi\left(t ; x_{0}, 0\right)\right| \leq \beta_{G A S}\left(\left|x_{0}\right|, t\right) .
$$

Thus 0-GAS holds, if, whenever the input $u$ is set to zero, the system (5) is globally asymptotically stable at $x=0$.

By results in [11] asymptotic gain and global asymptotic stability at 0 together are equivalent to ISS.

d) Problem description: Consider $n$ interconnected control systems given by

$$
\begin{gathered}
\dot{x}_{1}=f_{1}\left(x_{1}, \ldots, x_{n}, u\right) \\
\vdots \\
\dot{x}_{n}=f_{n}\left(x_{1}, \ldots, x_{n}, u\right)
\end{gathered}
$$

where $x_{i} \in \mathbb{R}^{N_{i}}, u \in \mathbb{R}^{L}$ and $f_{i}: \mathbb{R}^{\sum_{j=1}^{n} N_{j}+L} \rightarrow \mathbb{R}^{N_{i}}$ is continuous and Lipschitz in the first $n$ arguments uniformly with respect to $u$ for $i=1, \ldots, n$. Here $x_{i}$ is the state of the $i^{\text {th }}$ subsystem, and $u$ is considered as an external control variable.

We call the $i^{\text {th }}$ subsystem of (8) ISS, if for its solution $x_{i}(t)$ starting at $x_{i}(0)$, there exist functions $\beta_{i}$ of class $\mathcal{K} \mathcal{L}$ and $\gamma_{i j}, \gamma$ of class $\mathcal{K}$, such that

$$
\left|x_{i}(t)\right| \leq \beta_{i}\left(\left|x_{i}(0)\right|, t\right)+\sum_{j=1}^{n} \gamma_{i j}\left(\| x_{j[0, t]}||\right)+\gamma(\|u\|)
$$

for all $t \geq 0$ and all solutions. Note, that for notational simplicity we allow the case $\gamma_{i j} \equiv 0$ and require $\gamma_{i i} \equiv 0$ for all $i$. The functions $\gamma_{i j}$ and $\gamma$ are called (nonlinear) gains. We write $\Gamma:=\left(\gamma_{i j}\right)$ and define $\Gamma: \mathbb{R}_{+}^{n} \rightarrow \mathbb{R}_{+}^{n}$ by

$$
\Gamma\left(s_{1}, \ldots, s_{n}\right)^{T}:=\left(\sum_{j=1}^{n} \gamma_{1 j}\left(s_{j}\right), \ldots, \sum_{j=1}^{n} \gamma_{n j}\left(s_{j}\right)\right)^{T}
$$

for $s=\left(s_{1}, \ldots, s_{n}\right)^{T} \in \mathbb{R}_{+}^{n}$. We refer to $\Gamma$ as the gain matrix, although it might not be a linear mapping. Note that by the properties of $\gamma_{i j}$ for $s_{1}, s_{2} \in \mathbb{R}_{+}^{n}$ we have that $\Gamma$ defines a monotone mapping, i.e.,

$$
s_{1} \geq s_{2} \Rightarrow \Gamma\left(s_{1}\right) \geq \Gamma\left(s_{2}\right) .
$$

Assuming the subsystems to be ISS, we now like to ask, whether the whole system defined by

$$
x=\left(x_{1}^{T}, \ldots, x_{n}^{T}\right)^{T}, \quad f=\left(f_{1}^{T}, \ldots, f_{n}^{T}\right)^{T}
$$

and

$$
\dot{x}=f(x, u)
$$

is ISS (from $u$ to $x$ ).

\section{MAIN RESULTS}

In the following we present a nonlinear version of the small-gain theorem for networks and also a version for the case when the gains are linear functions.

1) Nonlinear gains: We need the following notation. For $\alpha_{i} \in \mathcal{K}_{\infty}, i=1, \ldots, n$ define $D: \mathbb{R}_{+}^{n} \rightarrow \mathbb{R}_{+}^{n}$ by

$$
D\left(s_{1}, \ldots, s_{n}\right)^{T}:=\left(\begin{array}{c}
\left(\operatorname{Id}+\alpha_{1}\right)\left(s_{1}\right) \\
\vdots \\
\left(\operatorname{Id}+\alpha_{n}\right)\left(s_{n}\right)
\end{array}\right) .
$$

Theorem 4 (small-gain theorem for networks): Consider system (8) and suppose that each subsystem is ISS, i.e., condition (9) holds for all $i=1, \ldots, n$. Let $\Gamma$ be given by (10). If there exists a mapping $D$ as in (13), such that

$$
(\Gamma \circ D)(s) \nsupseteq s, \quad \forall s \in \mathbb{R}_{+}^{n} \backslash\{0\},
$$

then the system (12) is ISS from $u$ to $x$.

Remark 5: Although looking very complicated to handle at first glance, condition (14) is a straightforward extension of the ISS small gain theorem of [5]. It has many interesting interpretations, as we will discuss in Section IV.

The following lemma provides an essential argument in the proof of Theorem 4, see [1] for details.

Lemma 6: Let $D$ be as in (13) and suppose (14) holds. Then there exists a $\varphi \in \mathcal{K}_{\infty}$ such that for all $u, v \in \mathbb{R}_{+}^{n}$,

$$
(\operatorname{Id}-\Gamma)(u) \leq v
$$

implies $|u| \leq \varphi(|v|)$.

We give an outline of the proof of Theorem 4 .

Proof: For finite times $t \geq 0$ and for $s \in \mathbb{R}_{+}^{n}$ we introduce the abbreviating notation

$$
\begin{aligned}
|x(t)| & :=\left(\left|x_{1}(t)\right|, \ldots,\left|x_{n}(t)\right|\right)^{T}, \\
\beta(s, t) & :=\left(\beta_{1}\left(s_{1}, t\right), \ldots, \beta_{n}\left(s_{n}, t\right)\right)^{T},
\end{aligned}
$$


and

$$
\gamma^{n}(\|u\|):=(\gamma(\|u\|), \ldots, \gamma(\|u\|))^{T} .
$$

The existence of a solution for (12) for all times can be easily checked using Lemma 6. Let

$$
s_{\infty}:=\varphi\left(\left|\beta(|x(0)|, 0)+\gamma^{n}(\|u\|)\right|\right),
$$

where $\varphi$ is given by Lemma 6 . This is a ultimate bound on the state $x(t)$.

To establish the ISS we utilize the same idea as in [5]: Instead of estimating $\left|x_{i}(t)\right|$ with respect to $\left|x_{i}(0)\right|$ in (9), we can also have the point of view that our trajectory started in $x_{i}(\tau)$ at time $0 \leq \tau \leq t$ and we followed it for some time $t-\tau$ and reach $x_{i}(t)$ at time $t$. For $\tau=t / 2$ this reads

$$
\begin{aligned}
\left|x_{i}(t)\right| & \leq \beta_{i}\left(\left|x_{i}(t / 2)\right|, t / 2\right)+\sum_{j \neq i} \gamma_{i j}\left(\| x_{i,[t / 2, t]}||\right)+\gamma(u) \\
& \leq \beta_{i}\left(s_{\infty}, t / 2\right)+\sum_{j \neq i} \gamma_{i j}\left(\| x_{i,[t / 2, \infty)}||\right)+\gamma(u)(19) \\
& =\tilde{\beta}_{i}\left(s_{\infty}, t\right)+\sum_{j \neq i} \gamma_{i j}\left(\| x_{i,[t / 2, \infty)}||\right)+\gamma(u) \quad(20)
\end{aligned}
$$

where we again applied (11) to obtain (19) and defined

$$
\tilde{\beta}_{i}\left(s_{i}, t\right):=\beta_{i}\left(s_{i}, t / 2\right)
$$

which is of class $\mathcal{K} \mathcal{L}$. To write inequality (20) in vector form, we define

$$
\tilde{\beta}(s, t):=\left(\tilde{\beta}_{1}\left(s_{1}, t\right), \ldots, \tilde{\beta}_{n}\left(s_{n}, t\right)\right)^{T}
$$

for all $s \in \mathbb{R}_{+}^{n}$. Denoting by $s_{\infty}^{n}:=\left(s_{\infty}, \ldots, s_{\infty}\right)^{T}$ we obtain the vector formulation of (20) as

$$
|x(t)| \leq \tilde{\beta}\left(s_{\infty}^{n}, t\right)+\Gamma \circ\left\|x_{[t / 2, \infty)}\right\|+\gamma^{n}(\|u\|) .
$$

By the boundedness of the solution we can take the upper limit on both sides of (22). By Lemma 3 we have

$$
\limsup _{t \rightarrow \infty}|x(t)|=\limsup _{t \rightarrow \infty}\left\|x_{[t / 2, \infty)}\right\|=: l(x),
$$

and it follows that

$$
(\operatorname{Id}-\Gamma) \circ l(x) \leq \gamma^{n}(\|u\|)
$$

since $\lim _{t \rightarrow \infty} \tilde{\beta}\left(s_{\infty}^{n}, t\right)=0$. Finally, by Lemma 6 we have

$$
|l(x)| \leq \varphi\left(\left|\gamma^{n}(|| u||)\right|\right)
$$

for some $\varphi$ of class $\mathcal{K}_{\infty}$. But (23) is the asymptotic gain property (6). Together with 0-GAS (the proof is omitted for brevity) this implies the ISS for (12) cf. [11, Theorem 1].

2) Linear gains: Suppose the gain functions $\gamma_{i j}$ are all linear, hence $\Gamma$ is a linear mapping and (10) is just matrixvector multiplication. Then we have the following

Corollary 7: Consider $n$ interconnected ISS systems as in the previous section on the problem description with a linear gain matrix $\Gamma$, such that for the spectral radius $\rho$ we have

$$
\rho(\Gamma)<1 .
$$

Then the system defined by (12) is ISS.
Remark 8: For the case of large-scale interconnected input-output systems a similar result exists, which can be found in a monograph by Vidyasagar, cf. [12, p. 110]. It also covers Corollary 7 as a special case. The condition on the spectral radius is quite the same, although it is applied to a test matrix, whose entries are finite gains of products of interconnection operators and corresponding subsystem operators. These gains are non-negative numbers and, roughly speaking, defined as the minimal possible slope of affine bounds on $|G(x)|_{L_{\infty}}$ with respect to $|x|_{L_{\infty}}$ for operators $G: L_{\infty} \rightarrow L_{\infty}$ and states $x \in L_{\infty}$.

Proof: The proof is essentially the same as for Theorem 4, but note that instead of Lemma 6 we now directly have existence of

$$
(\mathrm{Id}-\Gamma)^{-1}=\mathrm{Id}+\Gamma+\Gamma^{2}+\ldots
$$

since $\rho(\Gamma)<1$ and from the power sum expansion it is obvious that $(\mathrm{Id}-\Gamma)^{-1}$ is a non-decreasing mapping, i.e., for $d_{1}, d_{2} \geq 0$ we have $(\operatorname{Id}-\Gamma)^{-1}\left(d_{1}+d_{2}\right)-(\operatorname{Id}-\Gamma)^{-1}\left(d_{1}\right) \geq 0$.

Thus at the two places where Lemma 6 has been used we can simply apply $(\mathrm{Id}-\Gamma)^{-1}$ to get the desired estimates.

\section{A. Application to linear systems}

An important special case is, when the underlying systems are linear themselves. Consider the following setup, where in the sequel we omit the external input, formerly denoted by $u$, for notational simplicity. Let

$$
\dot{x}_{j}=A_{j} x_{j}, \quad x_{j} \in \mathbb{R}^{N_{j}}, \quad j=1, \ldots, n
$$

describe $n$ globally asymptotically stable linear systems, which are interconnected by the formula

$$
\dot{x}_{j}=A_{j} x_{j}+\sum_{k=1}^{n} \Delta_{j k} x_{k} \quad j=1, \ldots, n,
$$

which can be rewritten as

$$
\dot{x}=(A+\Delta) x,
$$

where $A$ is block diagonal, $A=\operatorname{diag}\left(A_{j}, j=1, \ldots, n\right)$, each $A_{j}$ is Hurwitz (i.e., the spectrum of $A_{j}$ is contained in the open left half plane) and the matrix $\Delta=\left(\Delta_{j k}\right)$ is also in block form and encodes the connections between the $n$ subsystems. We suppose that $\Delta_{j j}=0$ for all $j$. Define the matrix $R=\left(r_{j k}\right), R \in \mathbb{R}_{+}^{n \times n}$ by $r_{j k}:=\left\|\Delta_{j k}\right\|$. For each subsystem, there exist non-negative numbers $M_{j}, \lambda_{j}$, such that $e^{A_{j} t} \leq M_{j} e^{-\lambda_{j} t}$ for all $t \geq 0$.

Define a matrix $D \in \mathbb{R}_{+}^{n \times n}$ by $D:=\operatorname{diag}\left(\frac{M_{j}}{\lambda_{j}}, j=\right.$ $1, \ldots, n)$.

From the last subsection we obtain

Corollary 9: If $\rho(D \cdot R)<1$ then (27) is globally asymptotically stable.

Note that this is a special case of the theorem, which can be found in Vidyasagar [12, p. 110], see Remark 8.

Proof: Denote the initial value by $x^{0}$. Then by elementary ODE theory we have

$$
x_{j}(t)=e^{A_{j} t} x_{j}^{0}+\sum_{k \neq j} \int_{0}^{t} e^{A_{j}(t-s)} \Delta_{j k} x_{k}(s) d s
$$


and by standard estimates

$$
\left|x_{j}(t)\right| \leq M_{j} e^{-\lambda_{j} t}+\sum_{k \neq j} r_{j k} \frac{M_{k}}{\lambda_{k}}\left\|x_{k,[0, t]}\right\| .
$$

As one can see from (29), in this case the gain matrix happens to be $\Gamma=D \cdot R$.

It is noteworthy, that this particular corollary is also a consequence of a recent paper [6] by Hinrichsen, Karow and Pritchard. For the convenience of the reader we state a simplified version of one of their results here.

Let $\|\cdot\|_{R}$ denote the weighted maximum norm given by $\|\Delta\|_{R}:=\max _{(j, k): r_{j k} \neq 0} r_{j k}^{-1}\left\|\Delta_{j k}\right\|$. Let $\mathbb{B}_{R}:=\{B=$ $\left(B_{j k}\right)$ such that $r_{j k}=0$ implies $\left.B_{j k}=0\right\}$.

The stability radius $r(A, R)$ of the matrix $A$ with respect to the norm $\|\cdot\|_{R}$ is defined by

$$
\begin{array}{r}
r(A, R):=\inf \left\{\|B\|_{R} \mid B \in \mathbb{B}_{R}:\right. \\
\dot{x}=A x+B x \text { is unstable }\}
\end{array}
$$

The stability radius is characterized by the fact, that block perturbations below it cannot destabilize the system, whereas there is a block perturbation of size equal to $r(A, R)$ destabilizing the nominal system $A$. The matrix $R$ encodes which block entries may be non-zero at all.

This means, that whenever a block matrix $B=\left(B_{j k}\right)$ with the property

$$
r_{j k}=0 \text { implies } B_{j k}=0
$$

fulfills $\|B\|_{R}<r(A, R)$ then the system given by $\dot{x}=$ $A x+B x$ is stable (i.e., $\mathrm{A}+\mathrm{B}$ is Hurwitz).

Lemma 10: Let $A_{1}, \ldots, A_{n}$ be Hurwitz, i.e., $\sigma\left(A_{j}\right) \subset$ $\mathbb{C}_{-}:=\{z \in \mathbb{C}: \operatorname{Re} z<0\}$. Then the stability radius $r(A, R)$ of matrix $A$ with respect to the norm weighted by $R$ is given by

$$
r(A, R)=\left(\sup _{i s \in \mathbb{R}} \rho\left(R \cdot \operatorname{diag}\left(\left\|\left(s I-A_{j}\right)^{-1}\right\|,{ }_{j=1, \ldots, n}\right)\right)\right)^{-1} .
$$

Note that this is a stripped down version of Corollary 4.3(b) of [6].

An alternative proof for Corollary 9 using Lemma 10 is sketched in the following: Using the notation from [6], we get a weighted norm by the matrix $R$. We apply Lemma 10 to show that the stability radius with respect to the weighted norm given by $R$ of the system given by $\dot{x}=A x$ is greater than one.

By Lemma 5.5.2.2 of [3] we obtain $\left\|\left(s I-A_{j}\right)^{-1}\right\| \leq \frac{M_{j}}{\lambda_{j}}$ for $i s \in \mathbb{R}$. Hence $\rho(D \cdot R)<1$ implies

$$
\sup _{i s \in \mathbb{R}} \rho\left(R \cdot \operatorname{diag}\left(\| s I-A_{j}\right)^{-1} \|, j=1, \ldots, n\right)<1,
$$

hence $r(A, R)>1$, whereas $\|\Delta\|_{R}=1$, which proves the corollary.

\section{INTERPRETATION OF THE PREREQUISITES}

In this section we wish to provide insight into the small gain condition of Theorem 4 . We first show, that the result covers the known interconnection results for cascades and feedback interconnections. We then compare the condition with the linear case.
It is an easy consequence of Theorem 4, that an arbitrarily long feed forward cascade of ISS subsystems is ISS again. If the subsystems are enumerated consecutively and the gain function from subsystem $i$ to subsystem $j>i$ is denoted by $\gamma_{i j}$, then the resulting gain matrix has non-zero entries only below the diagonal. For arbitrary $\alpha \in \mathcal{K}_{\infty}$ the gain matrix with entries $\gamma_{i j} \circ\left(\operatorname{Id}_{\mathbb{R}_{+}}+\alpha\right)$ for $j>i$ and 0 for $j \leq i$ clearly satisfies (14). Therefore the feed forward cascade itself is ISS.

Consider $n=2$ in equation (8), i.e., two subsystems with linear gains. Then in Corollary 7 we have

$$
\Gamma=\left[\begin{array}{cc}
0 & \gamma_{12} \\
\gamma_{21} & 0
\end{array}\right], \quad \gamma_{i j} \in \mathbb{R}_{+}
$$

and $\rho(\Gamma)<1 \Leftrightarrow \gamma_{12} \gamma_{21}<1$. Hence we obtain the known linear small-gain theorem, which is also a special case of [4] and [2].

For nonlinear gains and $n=2$ the condition (14) in Theorem 4 reads as follows

$$
\left(\begin{array}{l}
\gamma_{12} \circ\left(\operatorname{Id}+\alpha_{2}\right)\left(s_{2}\right) \\
\gamma_{21} \circ\left(\operatorname{Id}+\alpha_{1}\right)\left(s_{1}\right)
\end{array}\right) \ngtr\left(\begin{array}{c}
s_{1} \\
s_{2}
\end{array}\right),
$$

for all $\left(s_{1}, s_{2}\right) \in \mathbb{R}_{+}^{2}$. This is easily seen to be equivalent to

$$
\gamma_{12} \circ\left(\operatorname{Id}+\alpha_{2}\right) \circ \gamma_{21} \circ\left(\operatorname{Id}+\alpha_{1}\right)(s)<s, \quad \forall s>0 .
$$

(Just check what happens for $\left(\gamma_{12} \circ\left(\operatorname{Id}+\alpha_{2}\right)\left(s_{2}\right), s_{2}\right)$, etc.) The latter is equivalent to the condition in the small-gain theorem of [5], which is

$$
\left(\operatorname{Id}+\tilde{\alpha}_{1}\right) \circ \gamma_{21} \circ\left(\operatorname{Id}+\tilde{\alpha}_{2}\right) \circ \gamma_{12}(s) \leq s, \quad \forall s>0
$$

for some other $\tilde{\alpha}_{1}, \tilde{\alpha}_{2} \in \mathcal{K}_{\infty}$ and all $s \in \mathbb{R}_{+}$, hence our theorem contains this result as a particular case.

We discuss some consequences from (14). Recall that for a non-negative matrix $\Gamma$ the following are equivalent:

(i) $\rho(\Gamma)<1$,

(ii) $\forall s \in \mathbb{R}_{+}^{n} \backslash\{0\}: \Gamma s \nsupseteq s$,

(iii) $\Gamma^{k} \rightarrow 0$, for $k \rightarrow \infty$,

(iv) there exist $a_{1}, \ldots, a_{n}>0$ such that $\forall s \in \mathbb{R}_{+}^{n} \backslash\{0\}$ : $\Gamma\left[I+\operatorname{diag}\left(a_{1}, \ldots, a_{n}\right)\right] s \nsupseteq s$.

Note that (iv) is the linear version of (14). As condition (i) is not useful in the nonlinear setting, we have turned to (ii). We found for the present approach that we need to relax (ii) to (iv) in the nonlinear case. In the nonlinear case (iv) implies (ii) but not vice versa. This may be seen by considering the case of two nonlinear subsystems for which we have to check (31). Let $\gamma_{12}=\operatorname{Id}_{\mathbb{R}_{+}}$and $\gamma_{21}(r)=r\left(1-e^{-r}\right)$. Since already $\lim _{r \rightarrow \infty}\left(\gamma_{12} \circ \gamma_{21}-\mathrm{Id}\right)(r)=0$ there certainly are no class $\mathcal{K}_{\infty}$ functions $\tilde{\alpha}_{i}, i=1,2$ such that (31) holds.

For the following statement let us define the open domains

$$
\Omega_{i}=\left\{x \in \mathbb{R}^{n}:\left|x_{i}\right|>\sum_{j \neq i} \gamma_{i j}\left(\left|x_{j}\right|\right)\right\} .
$$

Proposition 11: It follows from (14) that

$$
\Gamma(s) \nsupseteq s \quad \text { for any } \quad s \in \mathbb{R}_{+}^{n} \backslash\{0\} .
$$




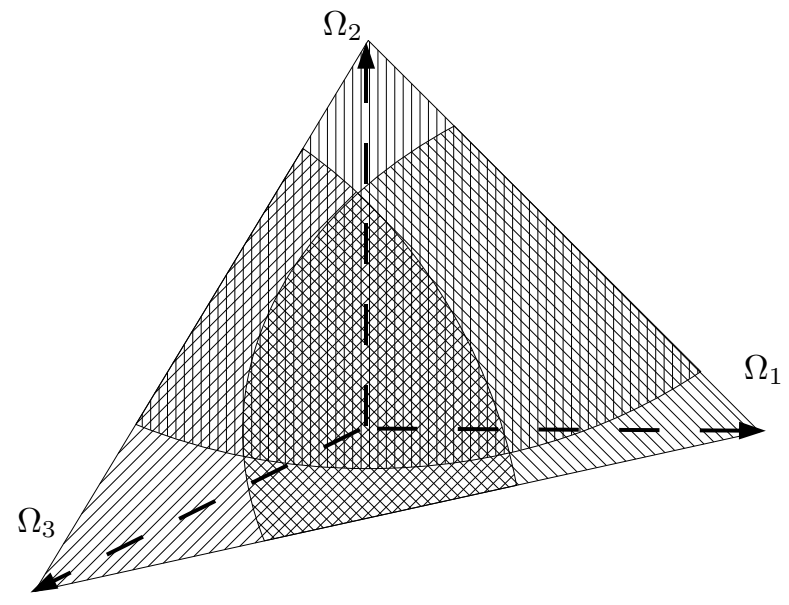

Fig. 3. Overlapping of $\Omega_{i}$ domains in $\mathbb{R}^{3}$

Property (32) also follows from the asymptotic stability of the system

$$
s(k+1)=\Gamma(s(k)), \quad k \in \mathbb{N}
$$

in $s^{*}=0$ (with state space $\mathbb{R}_{+}^{n}$ ).

Finally, (32) is equivalent to

$$
\bigcup_{i=1}^{n} \Omega_{i}=\mathbb{R}^{n} \backslash\{0\} \quad \text { and } \bigcap_{i=1}^{n} \Omega_{i} \neq \emptyset .
$$

For linear $\Gamma$ any of (14), (32), (33), (34) is equivalent to

$$
\rho(\Gamma)<1 \text {. }
$$

For a proof of the proposition we refer to [1], where also the following stronger statement is proved: If the system is irreducible (that is, the graph associated with the gain network is irreducible), then properties (32) and (33) are in fact equivalent. There the authors also state an example that the implication from (32) to (33) fails for non-irreducible systems.

Note that while in the linear case $\rho(\Gamma)<1$ is equivalent to the existence of an $s>0$ such that $\Gamma(s)<s$ (which is to say $\left.\bigcap_{i=1}^{n} \Omega_{i} \neq \emptyset\right)$, this is not the case for nonlinear $\Gamma$.

Let us briefly explain, why the overlapping condition (34) is interesting: From the theory of ISS-Lyapunov function it is known, that a system of the form (5) is ISS if and only if there exists a smooth Lyapunov function $V$ with the property

$$
|x| \geq \gamma(|u|) \Rightarrow \nabla V(x) f(x, u)<-W(|x|),
$$

for some $W \in \mathcal{K}$. In the case of our interconnected system this condition translates to the existence of Lyapunov functions $V_{i}$ for the subsystems $i=1, \ldots, n$ with the property

$$
\begin{array}{r}
\left|x_{i}\right| \geq \sum \gamma_{i j}\left(\left|x_{j}\right|\right)+\gamma(|u|) \\
\Rightarrow \nabla V_{i}\left(x_{i}\right) f_{i}(x, u)<-W_{i}\left(\left|x_{i}\right|\right),
\end{array}
$$

Now for $u=0$ the condition of (35) is simply, that $x \in \Omega_{i}$. Thus the overlapping condition states that in each point of the state space one of the Lyapunov functions of the subsystems is decreasing. It is an interesting problem if via this a Lyapunov function for the whole system may be constructed.

A typical situation in case of three one dimensional systems $\left(\mathbb{R}^{3}\right)$ is presented in Figure 3 on a plane crossing the positive semi axis. The three sectors are the intersections of the $\Omega_{i}$ with this plane.

\section{CONCLUSIONS}

We considered a composite system consisting of an arbitrary number of nonlinear arbitrarily interconnected subsystems, as they naturally arise as dynamical systems on networks.

For this general case we derived a large scale system version of the nonlinear small-gain theorem. For the special case of linear interconnection gains this is a special case of a known theorem, cf. [12, page 110]. Applications of the result to linear systems has been discussed. Many interesting questions remain open, for instance concerning the construction of Lyapunov functions.

\section{ACKNOWLEDGMENTS}

This research is funded by the German Research Foundation (DFG) as part of the Collaborative Research Centre 637 "Autonomous Cooperating Logistic Processes: A Paradigm Shift and its Limitations" (SFB 637).

\section{REFERENCES}

[1] S. Dashkovskiy, B. Rüffer, and F. Wirth. An ISS small-gain theorem for general networks. Berichte aus der Technomathematik 05-05, Zentrum für Technomathematik, Bremen, Germany, 2005.

[2] L. Grüne. Input-to-state dynamical stability and its Lyapunov function characterization. IEEE Trans. Automat. Control, 47(9):1499-1504, 2002.

[3] D. Hinrichsen and A. J. Pritchard. Mathematical Systems Theory I Modelling, State Space Analysis, Stability and Robustness. SpringerVerlag, Berlin, 2005.

[4] Z.-P. Jiang, I. M. Y. Mareels, and Y. Wang. A Lyapunov formulation of the nonlinear small-gain theorem for interconnected ISS systems. Automatica J. IFAC, 32(8):1211-1215, 1996.

[5] Z.-P. Jiang, A. R. Teel, and L. Praly. Small-gain theorem for ISS systems and applications. Math. Control Signals Systems, 7(2):95120, 1994.

[6] M. Karow, D. Hinrichsen, and A. Pritchard. Interconnected systems with uncertain couplings: explicit formulae for $\mu$-values, spectral value sets and stability radii. Preprint, Institute of Mathematics, Technische Universität Berlin, 2005.

[7] H. K. Khalil. Nonlinear Systems. Prentice-Hall, Inc., Upper Saddle River, NJ, 1996.

[8] D. D. Šiljak. Large-scale dynamic systems. North-Holland Publishing Co., New York, 1979.

[9] E. Sontag and A. Teel. Changing supply functions in input/state stable systems. IEEE Trans. Automat. Control, 40(8):1476-1478, 1995.

[10] E. D. Sontag. The ISS philosophy as a unifying framework for stability-like behavior. In Nonlinear control in the year 2000, Vol. 2 (Paris), volume 259 of Lecture Notes in Control and Inform. Sci., pages 443-467. Springer, London, 2001.

[11] E. D. Sontag and Y. Wang. New characterizations of input-to-state stability. IEEE Trans. Automat. Control, 41(9):1283-1294, 1996.

[12] M. Vidyasagar. Input-output analysis of large-scale interconnected systems, volume 29 of Lecture Notes in Control and Information Sciences. Springer-Verlag, Berlin, 1981. 